\title{
Tactics for Designing Probes to Explore Parents' Differing Perspectives on Family Technology Use
}

\author{
Eleanor Chin Derix \\ School of Computer Science, University of Technology Sydney, Australia, eleanor.derix@gmail.com \\ Tuck Wah Leong \\ School of Computer Science, University of Technology Sydney, Australia, tuckwah.leong@uts.edu.au
}

\begin{abstract}
Experiences of technology use in everyday family life can be complex. In particular, tensions can arise when parents have differing perspectives on their family's technology use. This paper describes design tactics we used to create a probe collection that successfully supported explorations of these differing perspectives, and to uncover the tensions involved whilst remaining sensitive to any existing conflict. The tactics created opportunities for conversation between parents and to shift their individual perspectives. These tactics helped to raise the awareness sets of parents' had of each other's perspectives on their family's technology use. Unexpected insights emerged that even surprised our participants, when they were asked to invert their point of view to imagine how their technologies might experience domestic life. Furthermore, deeper insights emerged when participants' responses to individual probes were viewed together, as a collection.
\end{abstract}

\section{CCS CONCEPTS}

- Human-centred computing $\bullet$ HCI design and evaluation methods

\section{KEYWORDS}

Probes, design tactics, family, parents, technology, experiences.

\section{Introduction}

This methods paper contributes to our understanding of how probes can be used to better understand some of the complex experiences associated with technology use within families $[6,27,36]$. Specifically, the differing individual perspectives within sets of parents, regarding their family's experiences of day-to-day technology use $[1,15,40]$. In particular, it describes how we designed a collection of probes using two design tactics to (i) create opportunities for conversation between sets of parents, and (ii) to shift the perspectives of our participants. These tactics were used to help explore the different experiences, attitudes and expectations of parents, uncovering the tensions involved, while remaining sensitive to any existing conflict. Our probes also enabled reflection of socially undesirable, uncomfortable and even hypocritical situations. Overall, this paper contributes to an approach of how probes can be designed and used to productively support explorations of individual and co-experiences of technology use within domestic life. We hope that the design tactics and overall approach presented in this paper can help encourage researchers' efforts to develop more productive research tools to support inquiries of domestic HCI and, more broadly, of co-experiences within social groups.

Also, we highlight the value of using these distinct design tactics in combination, within our probes collection, demonstrating how the cumulative learnings revealed richer, unexpected reflections when compared to the sum of the learnings derived from the use of each individual probe. We hope that the knowledge presented in this paper can add to researchers' efforts to develop more productive research tools to support inquiries of domestic HCI.

\section{Researching Experiences of Family Technology Use in $\mathrm{HCl}$}

The family is the fundamental unit of society [50]. Technology use has become very much woven into the fabric of family life as it has in society [38]. Meanwhile, uncertainty surrounds the potentially adverse effect it might have on children $[9,34,42]$ and family relationships $[8,23,40]$. This has led to various efforts within HCI to develop our understanding of how families experience technology use [19, 48, 49]. However, researching family technology use presents some challenges. For instance, the challenge of how to define 'family'. This requires researchers to be cognizant of a broad and diverse range of family compositions [19]. In exploring the design of technologies for families, HCI has tended to define family either as a unit of people living in a home together, or who are related to each other [31]. The privacy concerns involved when attempting to research intimate domestic spaces, especially when 
involving children [19] present additional challenges. Practical challenges are also presented, by the need to integrate research into the busy day-to-day lives of multiple family members e.g. due to work commitments of parents [55]. The challenges presented by researching everyday domestic experiences has inspired a diverse set of methodologies, often requiring the refinement or reinvention of investigative approaches [16]. These varied methods used in HCI research of families has included the use of surveys [25, 43], interviews [1, 6], observations [26, 40] participatory design [44, 47], prototype testing [10], speculative design [17] and probes [29, 53].

In their explorations of technology rules in families, Mazmanian and Lanette [40] remind us of the need for research tools that don't overlook the messiness of family life, the power differentials between individual family members (e.g., parents and children), the varying expectations between family members, and changing family contexts. Furthermore, [40] note the risk of participants (particularly parents) providing socially desirable responses. We believe that the use of probes may play a part in helping to alleviate some of these concerns.

\subsection{Probes to Explore Experiences of Family Technology Use}

Since Gaver et al. [21] introduced cultural probes to HCI, these playful and open-ended tools have been used effectively to explore elements of domestic life $[30,45,53]$. As well as stimulating early dialogue with participants, probes support reflection by users. The ambiguity associated with completing probes offers participants a sense of privacy that has allowed them to be utilized in sensitive settings, or with populations that require sensitivity [7]. Probes can, therefore, enable intimate and personal issues to be addressed $[12,35]$. Their ability to reveal experiential and emotional aspects of design has been well established [37].

Many HCI researchers have demonstrated the effective use of a dialogical approach with probes. This involves exploring aspects of participants' lives by offering them opportunities to express themselves through completed probes [39], in conjunction with contextual interviews. This approach of 'probing for empathy' rather than 'probing for inspiration' has been demonstrated effectively within families, as a means of encouraging participants to acknowledge experiences that might usually go unnoticed within everyday life [29]. This dialogical approach has also been particularly fruitful in exploring more ephemeral aspects of family experiences, such as intimacy [12, 13, 35].

One way in which researchers have used probes in research with families has been to design them as a joint family project, to be completed together in preparation for a family interview [29, 53]. Another approach has been to capture the perspectives of two individuals to explore a particular aspect of their relationship, e.g., studies on intimacy between couples [52] and between children and grandparents [13].

Isola and Fails' [32] review of HCI research involving families describes a tendency to design for individual family members, rather than families as a whole. It suggests that this risks promoting Turkle's [49] notion of 'being alone together'. That is why, some researchers are calling for more holistic approaches and ways to develop more holistic accounts of family experiences with technology [19,31]. As families' use of technologies become more pervasive, and as more technologies are brought into homes, it becomes critical that our understandings of family experiences with technologies are holistic and nuanced. This is a primary motivation behind the design of our probes.

\subsection{Parents' Complex Experiences of Family Technology Use}

Families comprise of individuals, but being a member of the family unit often involves reciprocity and a sense of common aspirations. This comes with expectations, duties, and responsibilities that usually depend on an individual's role within the family, and likely to change over time, for example, collaborating on pragmatic tasks like coordinating family activities or participating in leisure activities [13, 24]. They can also include establishing etiquette (e.g., routines, rituals) [6,35] and more nuanced, ephemeral acts (e.g., attention, affection, intimacy, and love) [52]. As technology use becomes more interwoven into the fabric of family life, technology can be seen to connect the living room with other worlds [38]. When it comes to technology use, individuals' different experiences, expectations, and attitudes may need to be balanced with those of other family members $[6,56]$.

Recently, research shows that a failure to balance and to negotiate between these diverse, sometimes conflicting outlooks of individual family members can lead to family tension and conflict $[6,15,56]$. In particular, tensions between parents is associated to family technology use, and to differing individual attitudes towards it [20,43]. Parents might have to negotiate contrasting approaches to implementing family technology rules, including towards how each other use technology $[1,15,43]$ as well as towards the parenting of their children's technology use [27, 51]. No wonder many parents come to associate everyday family technology use with complex, challenging experiences [15].

Efforts exploring the dynamics of family technology use have offered valuable glimpses into the complex experiences of parents with regards to family technology use. These efforts tend to focus on the experiences that mothers (e.g.[22]), or fathers (e.g.[2]) have with specific devices, such as smartphones (e.g [46]), activities, such as the use of social network sites, (e.g. [1]), or of particular times, such as mealtimes (e.g [43]). However, many researchers recognize the need to build a more holistic view of families' evolving experiences of technology use [19, 31]. This 
includes developing our understanding of the interplay between technology use and the complex dynamics within sets of parents. To do that, we require tools that can assist in exploring the different experiences of individual parents, regarding their family's technology use. Ideally, these tools would allow us to disentangle the individual perspectives within sets of parents, in a way that captures any associated conflict or tension while remaining sensitive to them. We anticipate that this involves being able to prompt parents to reflect on a wide range of positive, negative, neutral and ambiguous experiences with technology, as well as those that go almost unnoticed. Next, we will describe a set of three probes designed to address this challenge.

\section{Method: Probe Design and Deployment}

In their work with families, Isola and Fails [31] recommend that researchers should also consider the family as a group besides focusing on individual members. As such, we believe that Battarbee and Koskinen's [3] notion of coexperience, to attempt to understand both the individual and also the social user experience to be a useful theoretical concept to keep in mind when exploring family experiences. Desjardin et al.'s [16] review of HCI approaches to researching domestic experiences provides another source of inspiration for the design of our probes, suggesting researchers consider how different personal experiences of the same home might differ. They also propose considering the perspective of objects within the home, posing questions like, 'how does a fridge experience domestic life?' [16]. Guided by these recommendations, we considered ways to design a collection of probes that could support sets of parents to reflect upon the complexity that might exist within ordinary experiences of family technology use with a focus on surfacing and disentangling their individual perspectives. We found three different perspectives to explore (Fig. 1).

\section{Perspective 1 - The Self}

We want to encourage sets of parents to reflect upon their family's technology use. This includes each parent's individual perceptions both of their own use of technology and their family members technology use. This will often involve habitual or routine uses of technology given its prevalence within everyday life.

Perspective 2 - Relating the Self to Others

We also want to encourage sets of parents to reflect upon the relationships they have with their family members and the technologies commonly used within everyday family life. We hoped that guiding parents towards this perspective would enable us to explore individual perceptions within the same family.

Perspective 3 - Imagining Technology's Perspective

Finally, we wanted sets of parents to imagine how their technological devices might experience domestic life. This meant encouraging participants to reconsider their default point-of-view, 'the Self' to take ona different point-of-view. This was intended to promote reflections of the family unit; to surface more detached, candid consideration that may include any socially undesirable, or uncomfortable aspects.

\subsection{Designing Our Probes}

We designed three probes, (i) Family Experience Jar, (ii) Digital Family Tree, and (iii) Device Journal ('The Secret Life of Us'). Next, we describe how the design of each of our probes was intended to position the participant to reflect from these different perspectives.

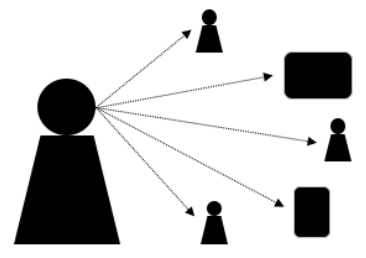

1: The Self

(Probe: Family Experience Jar)

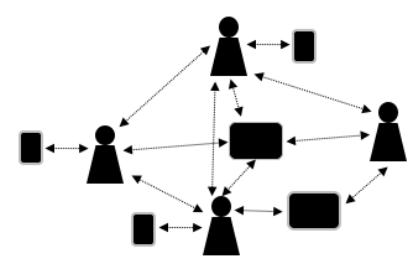

2: Relating to Others (Probe: Digital Family Tree )

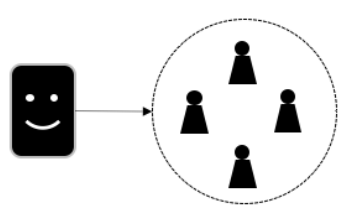

3: Technology's Perspective (Probe: Device Journal)

Figure 1. Exploring three perspectives to guide the design of probes

\subsubsection{Probe 1: Family Experience Jar.}

The Family Experience Jar probe (Fig. 2, left) is intended to encourage parents to log their individual experiences of everyday family technology use, from the perspective of 'The Self' (Fig. 1). We gave each set of parents a Jar and a pad of post-it notes to denote the type of experiences they have. Pink for recording positive experiences, blue for negative and yellow for experiences perceived to have both positive and negative aspects. We asked each parent to 
submit at least one note per day for the duration of the study, inviting them to make additional contributions as-andwhen such experiences occurred. Parents were not to discuss contributions with other each other. Finally, we asked each parent to initial and date their notes.

Aesthetics: By designing the Jars in an aesthetically pleasing way, we hoped to encourage parents to position them in visible locations in their homes, which might help remind them to make regular contributions.

Transparency: By choosing Jars made of clear glass, participants could see contributions amassing over time. The visible colour of the notes inside the Jar would also provide 'at-a-glance' idea of the types of experiences that had been logged. We hoped this might generate curiosity as to what the other parent had contributed; encouraging reflection and further participation.

Single Slot Opening: We cut a small slot into the lid of each Jar, meaning notes could only fit through if folded. Gluing the lid onto the Jar meant that notes could not be removed once they had been inserted. This prevented the details of each parent's notes being read by the other.

Size: We chose Jars large enough to contain several notes per day from each parent. We anticipated that visible empty space would promote more participation.

\subsubsection{Probe 2: Digital Family Tree.}

We designed the Digital Family Tree probe to explore parents' perceptions of relationships between technologies and their family. Each parent was asked to create an individual Family Tree diagram to illustrate the relationships between their family members and to include the technologies used in everyday family life (Fig. 3). During the first week of the study, each parent is asked to complete an individual Family Tree. During the second week, sets of parents are asked to compare their individual responses to the probe with each other. They are then asked to collaborate on a collective Family Tree (Fig. 2, middle). Including technologies into these diagrams was intended to play into people's tendency to anthropomorphize [18] and assist them to think about their family's relationships with technology differently. At the same time, we were interested in the differences between these perceptions and any resulting tensions. We explained that we would be especially interested in discussing how participants perceived the differences between individual Family Trees, and how they negotiated and collaborated to complete their shared Family Tree.

\subsubsection{Probe 3: Device Journal.}

The Device Journal probe (Fig. 2, right) was designed to encourage parents to completely reconsider their usual point-of-view and instead to take on the viewpoint of the technologies used in everyday family life. Inspired, in part, by artifact ecology [33], we devised a comic-style Journal called 'The Secret Life of Us', in which characters are technological devices, rather than humans.

We asked each parent to imagine how their devices experienced family life and individually journal them for two days. We hoped this playful probe would enable each parent to take a different viewpoint, with a refreshed perspective of their family and experiences. We hoped the tool could help surface insights of habitual technology use that might have been taken-for-granted, unremarkable, uncomfortable or even socially undesirable. Given the abstract nature of this task, we tried to support and inspire the participants by playing a short clip of 'Everything Is Alive'[11], a podcast series of fictional interviews with personified everyday objects, played by actors.

\subsection{Research Design and Probe Deployment}

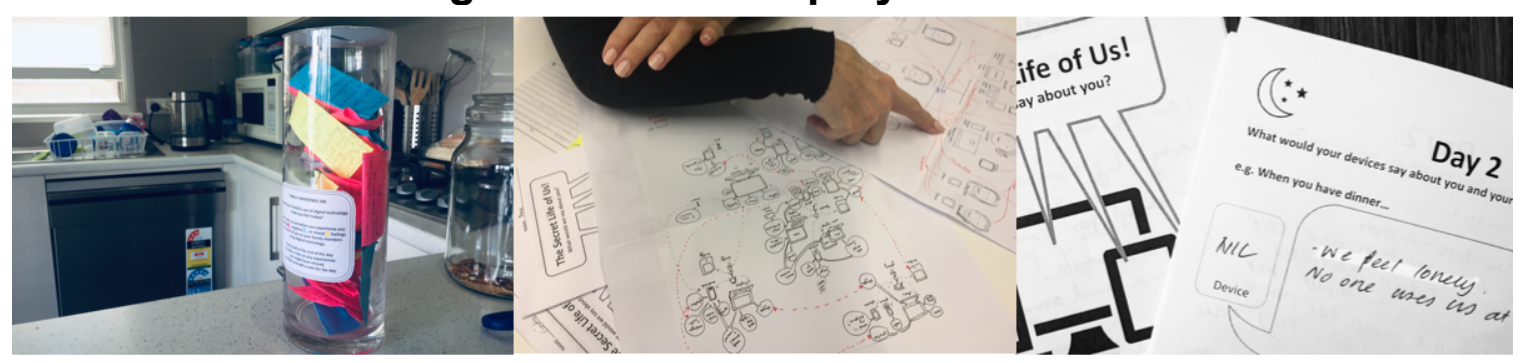

Figure 2. Probe Collection: Family Experience Jar (left), Digital Family Tree (middle), Device Journal (right)

This research was conducted in accordance with ethics approval from the University of Technology Sydney. Our study involved 17 parents of young children, from eight families. For each participant, the research spanned across 14 days.

On Day 1, we conducted an opening interview at each of the eight family homes. This lasted between 60-90 minutes. Each parent briefly introduced themselves and their family, and discussed technology use within broader family life, including routines, values, aspirations, and expectations. We then introduced parents to our probes and 
provided detailed instructions on how and when to complete them. We specified which probe activities were to be completed individually and which were to be completed collectively (Fig. 3).

They were told that they had 10-12 days to complete the probes. Between day 10-12, we collected completed probes and reviewed participants' responses, identifying interesting questions to be discussed during the closing interviews. On day 14 we held closing interviews with each of the 17 parents, individually. The choice to discuss the completed probes with each parent on their own, rather than with sets of parents, was a conscious one. We hoped it would encourage parents to be more candid and ensure that we captured their different perspectives. Each closing interview lasted between 50-70 minutes. This was a researcher-participant co-exploration of the completed probes, to make sense and to reflect, retrospectively, on their use of the probes. Also, this interview gave us the opportunity to seek clarifications of certain responses we found interesting when reviewing the completed probes.

\subsubsection{Participants.}

We recruited 17 parents to participate (P1-P17), from eight families with at least one child under the age of twelve. All parents had between one and three children, ranging between 1 month and 15 years. Participants held a range of occupations and a broad spectrum of outlooks and experience of technology. Participants were ethnically diverse. We should also note that one set of parents included three participants, a mother, aunty and grandmother, living together and raising three young children.

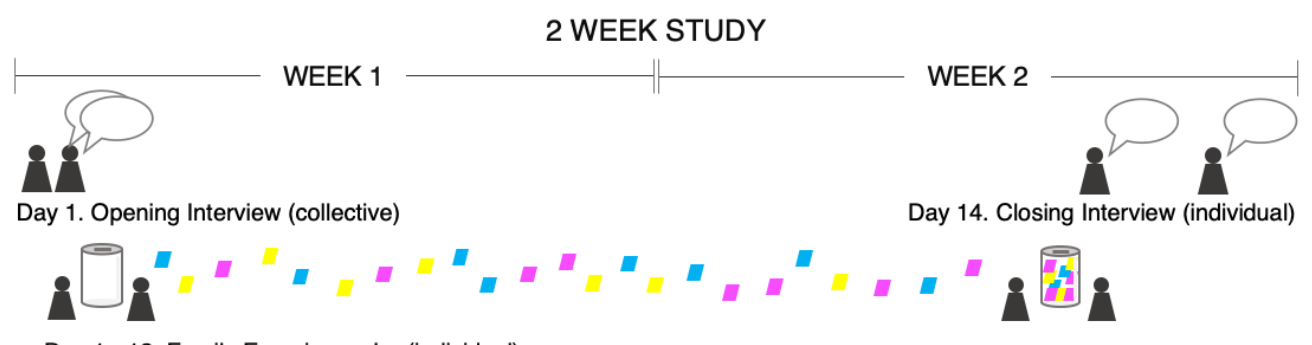

Day 1 - 12. Family Experience Jar (individual)

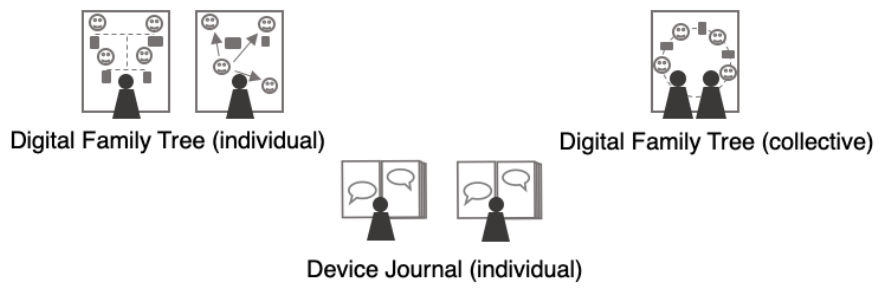

Figure 3. Probe deployment plan

\section{Findings: Effective Tactics for Designing Our Probes}

During the closing interviews, we found that our probe collection was successful in prompting varying levels of reflections about family experiences with technology. During our discussions, we heard many stories about the differing ways each parent perceived experiences of technology use within their family's everyday life. This included unexpected realizations that participants sometimes found to be emotional, and even surprising.

Within the messiness of family life, habitual, routine interactions with technology, and the experiences that result can seem automatic and inconsequential. Our probes were able to shift the perspectives of participants: for example, by inverting the conventional individual-centric point of view to imagine how particular technologies perceive family life. By reflecting from different perspectives, our participants began to interrogate aspects of their family's experiences and even reassess their views. The probes helped to reveal usually hidden experiences of family technology use, in particular, the way people perceive the role of technology, and the way it affects relationships within family life. The effectiveness of our probes was due to two distinct design tactics. The first tactic is to create opportunities for conversations. This means designing probes that can spur conversations between sets of parents, as well as selfreflections - internal conversations of the self. The other design tactic is to shift the perspectives of participants. This means designing probes that require sets of parents to see things from different perspectives, including each other's. Finally, our probes were found to be most effective when designed and put to work as a probe collection, combining these two distinct tactics. 


\subsection{Creating opportunities for conversations}

Our first tactic, of effectively creating opportunities for conversations, was employed in several ways through the design of our probe collection.

\subsubsection{Probes that create opportunities for internal dialogue.}

By asking participants to make regular contributions to the Experience Jar, we found that people became inadvertently more mindful of their technology use. Logging thoughts about their experiences of technology made people's internal dialogue explicit. This led them to be more aware of the relationships they have with technology which also led to realisations that were sometimes uncomfortable. For example, P13 left some notes in the jar that described her realisations about her technology use: "Wasting time! Realised surfing Facebook is a habit and not very satisfying...", "Frustrated that I keep almost compulsively) checking the weather app..." and "It has been a real struggle...not using technology as a babysitter" (P13, Jar).

When interviewed, P9 described how the Family Jar probe had resulted in some surprising realisations of her habits with technology. "I'd never really tied these automatic habits like just picking up your phone to an emotional motivation. What surprised me was thinking about the emotions around those experiences, rather than just going through the motions without really thinking about it." (P9, Int). We believe that had we not used this probe, it is unlikely that our participants would have the opportunity to recognise or question their more complicated relationships with technology.

We also used the Journal to prompt participants' internal dialogue. Over two days, participants were told to imagine how their devices would experience everyday domestic life.

Interestingly, most of our participants' journal entries consisted of what they imagined their devices would say to them. For example, P12 had imagined that before bed, his smartphone would tell him, "I can take you anywhere you want..." and "...let me help you sleep, and tomorrow I will wake you up again" (Journal). Such entries hint at the unspoken conversation or expectations participants have of their devices. They also reveal how much people felt dependent on their personal technologies.

When exploring these Journal entries during interviews, most participants further elaborated upon what their device would say. For example, when P15 described how she imagined her smartphone's experience of not being used would be, "It would be calling out to me; Use me! Use me more!" (Int). Encouraging participants to put themselves in their device's 'shoes' led some people to unwittingly reveal the lure they felt technology have, in particular, personal devices, like smartphones.

\subsubsection{Probes that allow sets of parents to compare their responses.}

We designed our probes to allow sets of parents to compare their individual responses with one another. This was done more subtly with the Family Experience Jar probe. While each parent was prevented from reading the details of what the other(s) had written (since notes had to be folded to fit through the Jar opening) the transparency of the Jar meant that the number and colour of the notes inside were visible. This enabled each parent to deduce the frequency and (positive, negative or ambivalent) nature of each other's experiences.

In the design of the Family Tree probe, the act of comparison was made more explicit. Sets of parents were asked to compare their individual Family Trees with one another, and to note any similarities or differences. In our interviews, we found that this aspect of the probe had enabled sets of parents to realise some of the assumptions they had made about their families' technology use. This allowed them to become more aware of each other's perspectives on technology use. For example, P12 was surprised to learn about the central role TV played in his family, realising that his family spent more time watching TV in his absence than he had previously imagined, "I see that the TV is central to the family, but I don't have any connection to it personally" (P12, Int). We noticed that several of our participants were surprised to learn that their assumptions about their families' technology use were not always right.

By comparing their individual Family Trees, some parents were prompted to re-examine family technology practices that they had previously taken for granted. For example, P2 who allows her children to access her phone had always assumed that her husband did the same. However, in her interview, she described her surprise at noticing that her husband's Family Tree showed no connection between his phone and the children. This had prompted her to ask her husband about this and learn for the first time that he did not, in fact, allow their children to use his phone since he considered it to be a work tool. In this way, we had designed a probe capable of creating opportunities for conversation between parents and raising awareness of different perspectives on technology use that tend to be overlooked in day-today family life.

\subsubsection{Probes that allow sets of parents to collaborate.}

After comparing individual Family Trees with each other, sets of parents were then asked to work together to create a collective Family Tree. By introducing collaboration into this probe, parents had to negotiate their individual 
perspectives on family technology use with each other. This created opportunities for a different kind of conversation, which we found, included interesting discussions, realisations, and challenges. Our participants told us that this probe led to some new-found realisations. For example, P8 explained how this task had spurred conversations within his family that led him to reassess the assumptions he had about their technology use: "I thought that it was a family desktop, but our Family Tree made me realise that it's really just me who uses it. I recognise now that these devices are more personal than shared" (Int). P9 discussed, "P10 will tell you a different story...I am surprised at P10's selfopinion of her own use. She doesn't think she uses (her smartphone) that much, but I really do. The (probes) gave me a legitimate lens to have a look at that." (P9, Int). This appreciation of the opportunities, provided by out probes, to discuss perceptions of technology use with one another, was also expressed by other participants.

\subsection{Shifting perspectives (using personification)}

Our first second tactic of shifting the perspectives of participants, was achieved through the design of probes that attempted to do this either explicitly or subtly.

\subsubsection{Explicit use of personification to shift perspectives.}

We used personification in the Device Journal probe to invert the human-centric view of seeing the world, by asking parents to journal experiences from the technology's point of view. Imagine how their devices might experience family life, to complete journal entries in the imagined voice of those devices.

Each journal required an introduction, in which individuals had to write about themselves in the third person, from the point of view of a device that would know them well. Almost all parents identified this device as their smartphone. When we read our participants' journal entries, we found that this probe activity revealed the strong agency these devices had in their lives. For instance, P13 imagined that her smartphone would write "I do everything for her" (P13, Journal). P7 had imagined what her smartphone would say of her, "She can't be separated from me" (P7, Journal). P1's had imagined that his smartphone would declare, "I am the center of his life!" (P1, Journal). Entries like this reveal how central the smartphone is in shaping the experiences of domestic life for many parents.

Using personification when designing this probe provided our participants with the opportunity to confer a character, a voice, opinion and a life to a technology. As a result, they were better able to reflect on their domestic lives from a different point of view. What we read were vivid and colorful descriptions of technologies, having relationships with individuals. Such accounts are not usually the kinds we often read or encounter in HCI. For instance, the imagined envy that one device would feel toward with another, "I'm a bit jealous that I have to sleep downstairs...the other phone gets to sleep in the bedroom and seems to get much more attention" (P13, Journal). Entries like these, highlight how personification can lead participants to inadvertently divulge clues about how technology use shapes family relationships.

During our discussions, parents explained how the 'inversion' of hearing what the device would say about them led them to new perspectives of themselves. This includes new realizations about their relationships with these technologies and with their family members, as well as the role these technologies had in their domestic life. Discussions of their journal entries also often triggered parents to reassess their relationships with their devices. For instance, P1 read a Journal entry aloud (written in the imagined voice of his smartphone), "I am a new addition to my Master's life" (P1, Journal). P1 looked at us and grinned, “Actually, it's probably the other way round!” (P1, Int). P7 also reassessed the relationship she had with her smartphone when discussing her one of her journal entries, "It would call me its mother...or maybe, actually, not a mother, a daughter. The phone is my mother. I am the daughter" (Int). For P13, "My smartphone is like a colleague, not a buddy - I'd go for a coffee with him, but not a beer!” (P13, Int).

Using personification gave license to people's imaginations and certainly added a sense of playfulness to this probe. It also helped surface a more detailed picture of participants' technology practices. In our interviews we noticed how some participants really enjoyed the task and injected humor into their responses. For instance, P15 laughed as she explained why she imagined her smartphone to be female, "It's too intelligent and sensitive to be male. It listens to me! It's is too organized (to be male)! It's addictive though. It distracts me from doing other things." (P15, Int). We also saw how effective personification was at freeing the imaginations of participants less eager to express their reliance on, and attachment to technology. For instance, asked about the relationship she had with her phone, P10 initially replied, "My imagination is struggling...I don't have that sort of relationship with my phone; it's just a thing" (P10, Int). When urged to consider an object that she felt more enthusiastically about, she conceded, "My bike would describe me as a hard taskmaster...but then, so would my phone, I reach for it compulsively. I feel physically anxious when the battery is low. I've never personified it before. It's a bit more of a boss, in that I must respond to it. I feel very apologetic if I stuff up something (e.g., miss an appointment by neglecting it)" (P10, Int).

Using personification in this probe helped to reveal emotional and ambivalent aspects of people's relationships with technology. In general, this allowed usually more concealed aspects of people's technology use to surface. As such, this 
probe reveals how people often take their relationships with technology for granted without explicitly reflecting upon it. These revelations would also have been much more challenging to pursue just using interviews.

\subsubsection{Subtle use of personification to shift perspectives.}

In the Family Tree probe, we asked parents to use a family tree diagram to illustrate relationships between family members and the technologies used in everyday family life. We felt that this is a more subtle form of personifying the technologies. Yet, we were still able to prompt valuable insights. This probe shifted our participants' perspectives (from the conventional view of 'the Self' to 'the Self in relation to others'), and also allowed them to rethink the role and relationships that technology has in family life.

When we asked our participants to review their completed Family Tree probe, they often compared the relationships between family members and devices, to relationships between family members. For instance, P8 pointed to how he had positioned his wife's smartphone between him and his wife when drawing his Family Tree, concluding, "her device probably knows more about her than I do." (P8, Int). His wife came to a similar conclusion in her interview, when she reviewed the way she had completed her own Family Tree, "my phone probably knows more about me than my family members" (P7, Int). Reflecting on Family Trees in this way revealed the surprising ways that technology use both mediates and shapes family relationships.

\subsection{Combining distinct tactics within a probe collection}

In designing our probes, we viewed them as a collection that would guide participants to look at family technology use from a range of different perspectives. By combining the responses to each probe, we hoped to not only build a more complete picture of individual perspectives on family technology use but to also build a more complete picture of the multiple perspectives that exist within families.

During our closing interviews, we asked our participants to reflect retrospectively on their experience of completing this probe collection. What they told us made us realise that by altering the perspective of our participants, and prompting them to detach and de-familiarise themselves from situations, people had begun to interrogate habitual behaviour that had been accepted as an inherent part of everyday family life. For example, P9 explained that the degree of conflict associated with her family's technology had become apparent to her as a result of completing the probes, "Overall (the probes) enabled me to reflect on all the conflict there is because of technology use. I guess I wasn't aware how much that was taking up my energy" (P9, Int).

In addition, when our participants reviewed their completed probes as a collection, they sometimes noticed contradictions in how they had responded to different probes. This challenged their preconceived ideas about their family's technology experiences. For example, P5 reacted to having a majority of pink notes in her Jar, "I expected more blue notes" (P5, Int). She considered this in conjunction with her responses to the Family Tree and Journal probes, which had raised her awareness of her ongoing efforts to limit her children's screen-time. Reflecting on her responses to the collection of probes, she deduced, "I guess I'm happy with the way we interact with technology...I'm more disturbed by the extent of it" (Int). What we found was by asking participants to review their probes as a collection, participants were able to consider their various responses at a more high-level and relational view, maybe even noting inconsistencies and mistaken assumptions about the role that technology plays in the lives of their families. In turn, this resulted in more nuanced reflections about the phenomenon.

\section{Discussion}

Our review of related literature acknowledges an established practice within HCI of using probes in a dialogical approach to support and stimulate discussions with participants in follow-up interviews when working with families e.g. [29, 30, 39]. We use probes in a similar fashion - as a dialogical tool to explore family experiences of technology.

This paper adds to HCI's scholarship by demonstrating how probes can be designed and used productively to support research inquiries, especially when seeking better understandings of technology use in families. We make this claim after examining our participants' responses to our probes, and after interviewing them about their use of our probes. Reflections of our findings have led to a number of methodoglocial insights. These insights pertain to the two distinct design tactics we have found to be effective when employed to design probes aimed at surfacing richer and more holistic understandings of family technology use. The three probes we designed, deployed and presented in this paper, exemplify how these distinct tactics can be combined and used successfully. First, we will reiterate why we need tools that can support researchers to better explicate the mutliple prespectives that surround technology use in families.

As many researchers remind us, families are not homogenous units but can be viewed as diverse communities with differences in age, gender and so on [29]. As such, the achievement of shared family aspirations requires the juggling of different individual roles, responsibilities, expectations, and attitudes. To achieve shared understandings within families requires compromise, negotiation, and reciprocity between individuals. Meanwhile, the increasing adoption 
and pervasive use of personal technologies in the domestic life of different individuals can and will continue to have significant effects on family dynamics [1, 6, 26, 27, 48]. As such, any HCI efforts to design digital technologies, in particular personal digital technologies that could be used in ways that are supportive of domestic dynamics will greatly benefit from deeper understandings of not only the different roles, but also the different and shared perspectives as well as attitudes of family members with regards to technology use in domestic life. However, this will also require effective tools to help surface and explicate the complex dynamics that surround family technology use. Asking questions through surveys and interviews may help, but there are also many aspects of family dynamics surrounding technology use that are not easily surfaced through these methods.

One challenge of exploring family experiences of technology is that people might not be totally aware of their own assumptions, approaches, and attitudes with regards to technology use. This is especially the case, with personal practices surrounding technology, in particular with personal technologies. This may be because technology use has become so habitual that individuals take them for granted. As a result, people are often unaware of their own perceptions, attitudes, and approaches to technology use. This leads people to make assumptions (whether accurately or not) about their own technology use and that of other family members. As illustrated in our findings section, many of our participants were surprised when confronted with unexpected realisations about themselves and also of other family members.

Another challenge is getting individuals to disclose the cause of tensions that might exist in their family as a result of technology use. Participants may find it uncomfortable or embarrassing to discuss private and possibly socially undesirable topics such as family conflict. The participants may not be fully aware of the underlying causes, or degree of the tension they experience. This is particularly true in families where tension around technology use has become an accepted part of domestic life.

Our work reveals the utility and effectiveness of using probes, or more specifically, probes that use particular tactics that can help surface and explore these challenging but important aspects of family dynamics surrounding technology use. Next, we discuss the two distinct tactics we used to design our probes.

\subsection{Tactic 1: Encouraging dialogue}

The first tactic we used when designing our probes was to create opportunities for conversation. This can be seen in various ways within our probe collection. In its core, these conversations are occasions for 'dialogue' (in Bakhtinian terms). For Bahktin, we are always in dialogue, not only with others and with everything in the world but also, internal conversations we have with ourselves [28]. Thus, this tactic can be seen in the design of probes that can make explicit individuals' internal dialogue. The Jar probe encouraged individuals to reflect upon their own technology use. This resulted in deeper awareness and greater (and sometimes uncomfortable) realisations about one's use and relationship with personal technologies. The Journal took a completely different approach by challenging individuals to rethink their relationships with their devices; asking them to reimagine the relationship and the agency their devices might have on their lives. This resulted in surprising and colourful reconceptualisations of the sometimes intimate and emotional relationships people have with their personal technologies. Their responses to these probes and the interviews also provided further insights into the individuals' dialogical sensemaking process with regards to their technology use [41].

This first tactic also involved the design of probes that make explicit one parent's relational sensemaking process to the other. In other words, surfacing how parents perceive and in turn, makes sense of their technology use in relation to one another. This approach was used in the Family Tree probe where sets of parents compared their own responses about technology use with responses from one another. This probe activity led to self-awareness and also an awareness (or at the very least, a consideration) of how one another perceives family technology use.

This first tactic is also seen in the design of probes that try to make explicit collaborative dialogical sensemaking $[37,41]$. The Family Tree probe involves sets of parents collaborating to complete an probe about their family's technology use. The probe aimed to surface both individual and shared perspectives. This probe reveals not only realisations of similarities, but also recognition of differences in perspectives, assumptions, and gave sets of parents insights into how one another made sense of their own technology use.

By designing our probes to engage sets of parents in activities to compare and talk about individual perspectives; to collaborate and to negotiate a common perspective, they were prompted to rethink the assumptions they had about each other. Using different approaches to provide opportunities for dialogue and collective sensemaking have surfaced discussions regarding the way technology use can trigger family conflict.

Of course, there are many other ways to provide opportunities for conversations. When designing probes, it will be helpful to think strategically on how to find ways to spur conversations; not only to help individuals to be aware of their own perspective towards technology use but also to surface their perceptions of how others in their family perceive and approach technology use. Finding productive ways to support families to explicate these different perspectives is crucial if we wish to develop a richer and more holistic understanding of family technology use. 
To the best of our knowledge, we not come across any explicit discussions in HCI of how probes can be designed strategically to support such explorations. Of course, there are many researchers who have used probes when researching families. Some designed probes for families to complete together [12, 29, 53]. For example, Horst et al.'s [29] probes, designed to explore empathy and to elicit inspiration from families, asked family members to work together to provide a single response. A follow-up interview was then conducted with all family members present to discuss their response. However, in their case, individual perspectives regarding empathy were absent. There are researchers working with families who have asked individuals to complete probes independently. For example, in studies of intimacy between couples [35, 52] and between children and their and grandparents [13], where individuals were asked to complete some of the probe activities independently. However, the interviews to explore the probe responses were conducted with the participants together, instead of separate interviews with individual participants. Their work did not seek to explicate differences of perspectives but sought agreements to inform designs. In that respect, potential tensions and differences between individual perspectives were not explored.

In this study, we found that these opportunities for conversations also benefitted families beyond the value they provide for researchers. For our families, having these conversations have helped to reduce assumptions and potential misunderstanding about technology use, that could lead to conflict.

\subsection{Tactic 2: Shifting perspectives}

The second tactic we used was to design probes that help to shift an individual's perspectives of experiences of technology use within everyday family life. We found this to be particularly useful when trying to explicate practices and attitudes surrounding technology use that have become habitual and taken for granted.

One effective approach to this tactic was to use the personification of personal devices. As we have described, the Device Journal asked people to give a voice and personality to their personal devices. Asking them to imagine how these devices would experience their family life was an effective strategy to shift (or even invert) the perspective of participants - from that of 'the self', to how an inanimate object such as their smartphones might experience their family life. This shift in perspective was able to reveal greater insights into roles, relationships and the agency that people ascribe to their personal technologies (e.g., smartphone as a mother-figure, TV as a peacekeeper), as well as the strong emotional pull their technologies seem to play, both in the lives of individuals and families.

We also attempted to shift our participants' perspective through the Family Tree probe. Here, the approach is to instigate a slightly subtler shift in perspective (when compared to the Device Journal). We accomplished this by tapping into people's natural tendency to anthropomorphise [18], asking individuals to consider their relationships with their devices, if these devices are seen as part of the family.

These two probes helped to free people's imagination and allowed them to rethink their relationships with their technologies. They are successful because our participants found these tasks to be playful and engaging. Our participants injected humour into their responses and provided all kinds of elaborate details such as the imagined feelings, relationships, and even gender that their technologies might have. More importantly, the probes were able to reveal surprising and unthought of realisations and insights for both researchers and participants. Many of our participants were surprised when they 'discovered' their routine, and habitual use of technology, through the voice of their technologies. As researchers, these probes encouraged our participants to disclose aspects of their families' technology use that they were less enthusiastic about, such as conflict and parenting challenges.

Researchers have used probes to get participants to see things in a new light. For example, Berkovich [5] asks people to imagine themselves at some point in the future to explore their financial goals. While Berkovich's approach guides participants to think about their finances in different ways, individuals remain in the point-of-view of 'the self' throughout the seven probe activities. As we explained, when exploring technology use, there is definitely value in ensuring that our understandings and inquiries shift beyond human-centric views. Only through gaining multiple perspectives (including that of our technologies) can we paint a more holistic picture of our complicated relationships with technologies, especially within domestic lives.

In HCI, defamiliarization has been offered as a useful strategy to help designers reimagine the design of domestic technologies [4]. However, we have not found any explicit discussions of how defamiliarization can be used productively to reveal hidden aspects of people's relationships and experiences with technologies. As Shklovsky [14] suggests, defamiliarization can provoke and refresh people's perception by heightening it through unfamiliarity and strangeness. By making something familiar and taken for granted (such as one's habitual use of personal technologies) strange, people are compelled to examine their automated perception. The Journal and the Family Tree were able, to a different extent, trigger reassessments that led to surprising realizations about their own practices and attitudes surrounding technology use.

On a side note, we also see the potential usefulness of using personification as a design tactic beyond our work. This design tactic has the capacity to prompt people to imagine the agency of technology, and to become aware of the 
potentially active and strongly emotional roles technologies can play in domestic life. As such, we posit that this tactic may very useful in explorations of domestic connected devices such as the IoT, and imaginations of how we can design future IoT devices that can be more supportive of family life.

\subsection{Combining tactics to understand the collective}

Finally, another methodological contribution we offer is to value of combining distinct tactics within a probe collection. Working with participants to review and consider their responses-comparing and contrasting responses they provided from one probe to the next, and as a whole collection, - shifted participants' perspectives of the phenomenon. This led to deeper reflections about technology use in their family because they can start to see patterns and inconsistencies. Designing a probe collection that combines two distinct tactics allowed insights to be built cumulatively and gradually from one probe to another, with the overall findings greater than the sum of the insights gathered from each probe.

While the use of probe collection is common within HCI [54], with the exception of [5], designing probe collections to intentionally combine two distinct tactics has not been explicitly described. Our work provides yet another example of how probes can be designed and used productively as a collection. [5] used a number of probes to guide participants to take different perspectives on a particular topic, and added the responses of each probe to "build a holistic understanding of the participant's perspective". We also used our probes to guide participants towards different perspectives. However, our probes were used to consider the different perspectives of individuals towards shared experiences. In addition, our probes were used to raise participants' awareness of the perspectives of other family members. While [5] uses a probe collection to build a better understanding of individuals, we use our probe collection to build a better understanding of not only individuals but also of the collective. This is because our probes enabled reflection on co-experiences and other family members' experiences with technology, even when our participants were absent.

Many researchers have used probes as a source of data triangulation. However, when designed, conceptualised and put to work as a set, the sum of the insights can add to probes' potential value for triangulation within research inquiry [53]. That is why we have a newfound appreciation for the value of designing and putting probes to work as a collection, rather than viewing probes as a series of separate artefacts used to capture fragmented aspects of a phenomenon of interest. We recommend that this approach is considered in any research involving the use probes, not only when exploring families.

\section{Limitations and Recommendations}

Our work has demonstrated the utility of a probe collection to effectively explore the differing perspectives within sets of parents, on their family's technology use. This collection is designed to both encourage conversation between sets of parents, whilst shifting their perspectives through the use of personification. This requires a reflective and skilled designer/researcher, able to conceptualise how individual probes can be designed to work synergistically, to elicit insights that are greater than the sum of their parts. It also requires probes to be deployed strategically to allow a combination of individual and collective responses to be captured. While probes that use of personification, to shift the perspective of participants, can be insightful, their abstract nature may demand a certain level of imagination of participants. Mindful of this, we suggest the need to support participants by providing some type of scaffolding material, in our case, the use of a podcast. When designing a probe collection, these more abstract probe activities should be preceded by probe activities that require less imagination and provide an easy entry point. While this method helped to reveal deeper understandings of parent's perspectives on family technology use, we suggest further work into how to utilise such design tactics to explore the experiences of all family members, including children, and wider social groups such as within workplaces.

\section{REFERENCES}

[1] Tawfiq Ammari, Priya Kumar, Cliff Lampe and Sarita Schoenebeck. 2015. Managing Children's Online Identities: How Parents Decide What to Disclose About Their Children Online. Proceedings of the 33rd Annual ACM Conference on Human Factors in Computing Systems. ACM, 1895-1904. [2] Tawfiq Ammari and Sarita Schoenebeck. 2015. Understanding and Supporting Fathers and Fatherhood on Social Media Sites. Proceedings of the 33rd Annual ACM Conference on Human Factors in Computing Systems. ACM, 1905-1914.

[3] Katja Battarbee and Ilpo Koskinen. 2005. Co-Experience: User Experience as Interaction. CoDesign 1, 1, 5-18.

[4] Genevieve Bell, Mark Blythe and Phoebe Sengers. 2005. Making by Making Strange: Defamiliarization and the Design of Domestic Technologies. ACM Trans. Comput.-Hum. Interact. 12, 2, 149-173. http://dx.doi.org/10.1145/1067860.1067862.

[5] Marianne Berkovich. 2009. Perspective Probe: Many Parts Add up to a Whole Perspective. CHI '09 Extended Abstracts on Human Factors in Computing Systems. ACM, Boston, MA, USA, 2945-2954. http://dx.doi.org/10.1145/1520340.1520422.

[6] Lindsay Blackwell, Emma Gardiner and Sarita Schoenebeck. 2016. Managing Expectations: Technology Tensions among Parents and Teens. Proceedings of the 19th ACM Conference on Computer-Supported Cooperative Work \& Social Computing. ACM, San Francisco, California, USA, 13901401. http://dx.doi.org/10.1145/2818048.2819928. 
[7] Kirsten Boehner, Janet Vertesi, Phoebe Sengers and Paul Dourish. 2007. How HCI Interprets the Probes. Proceedings of the SIGCHI Conference on Human Factors in Computing Systems. ACM, San Jose, California, USA, 1077-1086. http://dx.doi.org/10.1145/1240624.1240789.

[8] Danah Boyd. 2014. It's Complicated: The Social Lives of Networked Teens. Yale University Press.

[9] Ari Brown. 2011. Media Use by Children Younger Than 2 Years. Pediatrics 128, 5, 1040-1045.

[10] Barry Brown, Alex S. Taylor, Shahram Izadi, Abigail Sellen, Joseph Jofish' Kaye and Rachel Eardley. 2007. Locating Family Values: A Field Trial of the Whereabouts Clock. Springer Berlin Heidelberg, 354-371.

[11] Ian Chillag. 2018. Everything Is Alive. PRX. Radiotopia. Oakland, CA, USA.

[12] Thomas Dalsgaard, Mikael B. Skov, Malthe Stougaard and Bo Thomassen. 2006. Mediated Intimacy in Families: Understanding the Relation between Children and Parents. Proceedings of the 2006 conference on Interaction design and children. ACM, Tampere, Finland, 145-152. http://dx.doi.org/10.1145/1139073.1139110.

[13] Hilary Davis, Mikael B. Skov, Malthe Stougaard and Frank Vetere. 2007. Virtual Box: Supporting Mediated Family Intimacy through Virtual and Physical Play. Proceedings of the 19th Australasian conference on Computer-Human Interaction: Entertaining User Interfaces. ACM, Adelaide, Australia, 151-159. http://dx.doi.org/10.1145/1324892.1324920.

[14] Robert Con Davis. 1986. Contemporary Literary Criticism: Modernism through Poststructuralism. Longman Publishing Group.

[15] Eleanor Chin Derix and Tuck Wah Leong. 2018. Days of Our Lives: Family Experiences of Digital Technology Use. Proceedings of the 30th Australian Conference on Computer-Human Interaction. ACM, Melbourne, Australia, 332-337. http://dx.doi.org/10.1145/3292147.3292185.

[16] Audrey Desjardins, Ron Wakkary and William Odom. 2015. Investigating Genres and Perspectives in HCI Research on the Home. Proceedings of the 33rd Annual ACM Conference on Human Factors in Computing Systems. ACM, Seoul, Republic of Korea, 3073-3082.

http://dx.doi.org/10.1145/2702123.2702540.

[17] Abigail Durrant, Alex S. Taylor, David Frohlich, Abigail Sellen and David Uzzell. 2009. Photo Displays and Intergenerational Relationships in the Family Home. Proceedings of the 23rd British HCI Group Annual Conference on People and Computers: Celebrating People and Technology. British Computer Society, Cambridge, United Kingdom, 10-19.

[18] Nicholas Epley, Adam Waytz and John T Cacioppo. 2007. On Seeing Human: A Three-Factor Theory of Anthropomorphism. Psychological review $114,4,864$.

[19] Jerry Fails, Mona Leigh Guha, Michael Horn and Sara Isola. 2012. Technology for Today's Family. CHI '12 Extended Abstracts on Human Factors in Computing Systems. ACM, Austin, Texas, USA, 2739-2742. http://dx.doi.org/10.1145/2212776.2212709.

[20] Hasan Shahid Ferdous, Bernd Ploderer, Hilary Davis, Frank Vetere and Kenton O'Hara. 2015. Pairing Technology and Meals: A Contextual Enquiry in the Family Household. Proceedings of the Annual Meeting of the Australian Special Interest Group for Computer Human Interaction. ACM, Parkville, VIC, Australia, 370-379. http://dx.doi.org/10.1145/2838739.2838780.

[21] Bill Gaver, Tony Dunne and Elena Pacenti. 1999. Design: Cultural Probes. Interactions 6, 1, 21-29. http://dx.doi.org/10.1145/291224.291235

[22] Lorna Gibson and Vicki L Hanson. 2013. Digital Motherhood: How Does Technology Help New Mothers? Proceedings of the SIGCHI conference on human factors in computing systems. ACM, 313-322.

[23] Ellie Harmon and Melissa Mazmanian. 2013. Stories of the Smartphone in Everyday Discourse: Conflict, Tension \&\#38; Instability. Proceedings of the SIGCHI Conference on Human Factors in Computing Systems. ACM, Paris, France, 1051-1060. http://dx.doi.org/10.1145/2470654.2466134.

[24] Richard Harper. 2006. Inside the Smart Home. Springer Science \& Business Media.

[25] Alexis Hiniker, Sarita Y Schoenebeck and Julie A Kientz. 2016. Not at the Dinner Table: Parents' and Children's Perspectives on Family Technology Rules. Proceedings of the 19th ACM Conference on Computer-Supported Cooperative Work \& Social Computing. ACM, 1376-1389.

[26] Alexis Hiniker, Kiley Sobel, Hyewon Suh, Yi-Chen Sung, Charlotte P Lee and Julie A Kientz. 2015. Texting While Parenting: How Adults Use Mobile Phones While Caring for Children at the Playground. Proceedings of the 33rd annual ACM conference on human factors in computing systems. ACM, 727-736.

[27] Alexis Hiniker, Hyewon Suh, Sabina Cao and Julie A. Kientz. 2016. Screen Time Tantrums: How Families Manage Screen Media Experiences for Toddlers and Preschoolers. Proceedings of the 2016 CHI Conference on Human Factors in Computing Systems. ACM, San Jose, California, USA, 648660. http://dx.doi.org/10.1145/2858036.2858278.

[28] Michael Holquist. 2003. Dialogism: Bakhtin and His World. Routledge.

[29] Willem Horst, Ties Bunt, Stephan Wensveen and Lisa Cherian. 2004. Designing Probes for Empathy with Families. Proceedings of the conference on Dutch directions in HCI. ACM, Amsterdam, The Netherlands, 15. http://dx.doi.org/10.1145/1005220.1005239.

[30] Hilary Hutchinson, Wendy Mackay, Bo Westerlund, Benjamin B. Bederson, Allison Druin, Catherine Plaisant, Michel Beaudouin-Lafon, St, \#233, phane Conversy, Helen Evans, Heiko Hansen, Nicolas Roussel, Bj, \#246, rn Eiderb, \#228 and ck. 2003. Technology Probes: Inspiring Design for and with Families. Proceedings of the SIGCHI Conference on Human Factors in Computing Systems. ACM, Ft. Lauderdale, Florida, USA, 17-24. http://dx.doi.org/10.1145/642611.642616.

[31] Sara Isola and Jerry Alan Fails. 2012. Family and Design in the IDC and CHI Communities. Proceedings of the 11th International Conference on Interaction Design and Children. ACM, Bremen, Germany, 40-49. http://dx.doi.org/10.1145/2307096.2307102.

[32] Sara Isola and Jerry Alan Fails. 2012. Family and Design in the IDC and CHI Communities. Proceedings of the 11th International Conference on Interaction Design and Children. ACM, 40-49.

[33] Heekyoung Jung, Erik Stolterman, Will Ryan, Tonya Thompson and Marty Siegel. 2008. Toward a Framework for Ecologies of Artifacts: How Are Digital Artifacts Interconnected within a Personal Life? Proceedings of the 5th Nordic conference on Human-computer interaction: building bridges. 201-210.

[34] Nicholas Kardaras. 2016. Glow Kids: How Screen Addiction Is Hijacking Our Kids-and How to Break the Trance. St. Martin's Press.

[35] Jesper Kjeldskov, Martin Gibbs, Franks Vetere, Steve Howard, Sonja Pedell, Karen Mecoles and Marcus Bunyan. 2004. Using Cultural Probes to Explore Mediated Intimacy. Australasian Journal of Information Systems 11, 2.

[36] Priya Kumar and Sarita Schoenebeck. 2015. The Modern Day Baby Book: Enacting Good Mothering and Stewarding Privacy on Facebook. Proceedings of the 18th ACM Conference on Computer Supported Cooperative Work \& Social Computing. ACM, $1302-1312$.

[37] Tuck Wah Leong, Peter Wright, Frank Vetere and Steve Howard. 2010. Understanding Experience Using Dialogical Methods: The Case of Serendipity. Proceedings of the 22nd Conference of the Computer-Human Interaction Special Interest Group of Australia on Computer-Human Interaction. ACM, 256-263.

[38] S. Livingstone and E. Helsper. 2007. New Media \& Society 9, 4, 671.

[39] Tuuli Mattelmäki. 2006. Design Probes. Aalto University.

[40] Melissa Mazmanian and Simone Lanette. 2017. Okay, One More Episode: An Ethnography of Parenting in the Digital Age. Proceedings of the 2017 ACM Conference on Computer Supported Cooperative Work and Social Computing. ACM, 2273-2286.

[41] John McCarthy and Peter Wright. 2004. Technology as Experience. interactions 11, 5, 42-43.

[42] Brandon T. McDaniel and Jenny S. Radesky. 2018. Technoference: Longitudinal Associations between Parent Technology Use, Parenting Stress, and Child Behavior Problems. Pediatric Research. http://dx.doi.org/10.1038/s41390-018-0052-6.

[43] Carol Moser, Sarita Y Schoenebeck and Katharina Reinecke. 2016. Technology at the Table: Attitudes About Mobile Phone Use at Mealtimes. Proceedings of the 2016 CHI Conference on Human Factors in Computing Systems. ACM, 1881-1892.

[44] Carman Neustaedter and AJ Bernheim Brush. 2006. Linc-Ing the Family: The Participatory Design of an Inkable Family Calendar. Proceedings of the SIGCHI conference on Human Factors in computing systems. ACM, 141-150.

[45] Doenja Oogjes, William Odom and Pete Fung. 2018. Designing for an Other Home: Expanding and Speculating on Different Forms of Domestic

Life. Proceedings of the 2018 Designing Interactive Systems Conference. ACM, Hong Kong, China, 313-326.

http://dx.doi.org/10.1145/3196709.3196810. 
[46] Leysia Palen and Amanda Hughes. 2007. When Home Base Is Not a Place: Parents' Use of Mobile Telephones. Personal Ubiquitous Comput. 11, 5, 339-348. http://dx.doi.org/10.1007/s00779-006-0078-3.

[47] Catherine Plaisant, Aaron Clamage, Hilary Browne Hutchinson, Benjamin B Bederson and Allison Druin. 2006. Shared Family Calendars:

Promoting Symmetry and Accessibility. ACM Transactions on Computer-Human Interaction (TOCHI) 13, 3, 313-346.

[48] Diane J. Schiano, Christine Burg, Anthony Nalan Smith and Florencia Moore. 2016. Parenting Digital Youth: How Now? Proceedings of the 2016

CHI Conference Extended Abstracts on Human Factors in Computing Systems. ACM, San Jose, California, USA, 3181-3189.

http://dx.doi.org/10.1145/2851581.2892481.

[49] Sherry Turkle. 2017. Alone Together: Why We Expect More from Technology and Less from Each Other. Hachette UK.

[50] UN. 1995. Report of the World Summit for Social Development*. 1995, Report of the World Summit for Social Development.

[51] Elizabeth A. Vandewater, Seoung-Eun Park, Xuan Huang and Ellen A. Wartella. 2005. "No-You Can't Watch That": Parental Rules and Young

Children's Media Use. The American Behavioral Scientist 48, 5, 608-623.

[52] Frank Vetere, Martin R Gibbs, Jesper Kjeldskov, Steve Howard, Florian'Floyd' Mueller, Sonja Pedell, Karen Mecoles and Marcus Bunyan. 2005.

Mediating Intimacy: Designing Technologies to Support Strong-Tie Relationships. Proceedings of the SIGCHI conference on Human factors in

computing systems. ACM, 471-480.

[53] Amy Voida and Elizabeth D. Mynatt. 2005. Conveying User Values between Families and Designers. CHI '05 Extended Abstracts on Human

Factors in Computing Systems. ACM, Portland, OR, USA, 2013-2016. http://dx.doi.org/10.1145/1056808.1057080

[54] Jayne Wallace, John McCarthy, Peter C. Wright and Patrick Olivier. 2013. Making Design Probes Work. Proceedings of the SIGCHI Conference on

Human Factors in Computing Systems. ACM, Paris, France, 3441-3450. http://dx.doi.org/10.1145/2470654.2466473.

[ 55] Anne Weibert and Kai Schubert. 2010. How the Social Structure of Intercultural Computer Clubs Fosters Interactive Storytelling. Proceedings of

the 9th International Conference on Interaction Design and Children. ACM, Barcelona, Spain, 368-371. http://dx.doi.org/10.1145/1810543.1810616

[56] Sarita Yardi and Amy Bruckman. 2011. Social and Technical Challenges in Parenting Teens' Social Media Use. Proceedings of the SIGCHI

Conference on Human Factors in Computing Systems. ACM, 3237-3246. 